\title{
GCU
}

Glasgow Caledonian

University

University for the Common Good

\section{An underwater routing protocol with void detection and bypassing capability}

Ghoreyshi, Seyed Mohammad; Shahrabi-Farahani, Alireza; Boutaleb, Tuleen

Published in:

IEEE 31st International Conference on Advanced Information Networking and Applications (AINA), 2017

DOI:

10.1109/AINA.2017.82

Publication date:

2017

Document Version

Author accepted manuscript

Link to publication in ResearchOnline

Citation for published version (Harvard):

Ghoreyshi, SM, Shahrabi-Farahani, A \& Boutaleb, T 2017, An underwater routing protocol with void detection and bypassing capability. in IEEE 31st International Conference on Advanced Information Networking and Applications (AINA), 2017. Proceedings - International Conference on Advanced Information Networking and Applications, AINA, IEEE, pp. 530-537, The 31st IEEE International Conference on Advanced Information Networking and Applications, Taipei, Taiwan, 27/03/17. https://doi.org/10.1109/AINA.2017.82

\section{General rights}

Copyright and moral rights for the publications made accessible in the public portal are retained by the authors and/or other copyright owners and it is a condition of accessing publications that users recognise and abide by the legal requirements associated with these rights.

Take down policy

If you believe that this document breaches copyright please view our takedown policy at https://edshare.gcu.ac.uk/id/eprint/5179 for details of how to contact us. 


\title{
An Underwater Routing Protocol with Void Detection and Bypassing Capability
}

\author{
Seyed Mohammad Ghoreyshi, Alireza Shahrabi, and Tuleen Boutaleb \\ School of Engineering and Built Environment \\ Glasgow Caledonian University \\ Glasgow, UK \\ \{Seyed.MohammadGhoreyshi, A.Shahrabi, T.Boutaleb\}@gcu.ac.uk
}

\begin{abstract}
The development of routing protocols for underwater sensor networks (UWSNs) faces with different challenges, the most notable of which is perhaps how to deal with a void communication area. Over the past few years, some underwater protocols equipped with the void-handling techniques have been proposed to address this issue. The majority of them; however, are unable to detect and bypass the trapped nodes which can also lead a packet to become stuck in a void node. In this paper, we propose a new stateless routing protocol, called Energy-efficient and Void Avoidance Depth Based Routing (EVA-DBR) protocol which uses different control packets to detect the void and trapped nodes locally in the different area of network topology and then to exclude them from the routing paths using a passive participation approach. To the best of our knowledge, EVA-DBR is the first fully stateless routing protocol which can locally detect and bypass the trapped nodes in addition to the void nodes in UWSNs. The updating phase for sending the control packets is performed with the least possible cost, by eliminating the need for ACK packets. As an opportunistic routing protocol, EVADBR employs an adaptive forwarding area which can be resized and replaced according to the density and placement of the forwarding candidate nodes to enhance the energy efficiency and reliability. The results of our extensive simulation study indicate that EVA-DBR outperforms other protocols regarding the routing performance metrics.
\end{abstract}

\section{INTRODUCTION}

Nowadays, underwater sensor networks (UWSNs) has become a supportive means of ocean monitoring and resource discovery. Underwater sensor nodes are deployed in different depths of the region of interest to collect aquatic information and forward them to any one of the sinks on the surface [1], [2]. Sink nodes then deliver the accumulated information to the monitoring centre via the terrestrial radio links for further analysis, as shown in Fig. 1. Each underwater sensor node takes advantage of the acoustic transmission due to restrictions on the use of radio waves in the underwater environment. Each node also is equipped with a pressure gauge to measure its depth when deployed in the water [3]-[7]. Underwater routing protocols are aimed to improve the packet delivery with minimum cost in UWSNs in which greedy routing protocols are the most prominent approaches [2].

In greedy routing protocols, there is no need to discover and maintain the full path from the source node to the destination which makes them scalable to be used in the large networks with many nodes. Instead, only the information of one-hop or two hops is maintained in each node which eliminates the need for updating the long route path via the high overhead routing tables and routing messages [2]. However, in some cases, greedy forwarding may fail because the forwarding node cannot find any qualified node with a positive advancement towards the destination, so the packet is dropped even though there is a valid path from the sender to the destination. This phenomenon is called the communication void or local maximum [8].

For terrestrial sensor networks, greedy routing protocols seem fully mature and efficient to handle void area [8]. However, these protocols are quite impractical to be applied directly in the underwater environment, due to the different characteristics of UWSNs such as three-dimensionality, nodes mobility, low available bandwidth, slow propagation speed, ineffectiveness of Global Positioning System (GPS), energy constraints, and a lossy environment [1], [9]. Therefore, designing an efficient void-handling technique to improve the greedy routing protocol efficiency in UWSNs is crucial.

Several routing protocols have been proposed for UWSNs over the past few years. In some of these protocols, the void nodes can be detected and avoided thoroughly; however, few of them can deal with the trapped nodes issue. The trapped nodes are those that involving them in packet forwarding leads a packet to terminate in a void node [4], [7]. An efficient stateless routing protocol should proactively discover the trapped nodes in a preprocessing phase and avoid them during the packet forwarding.

Motivated by the above considerations, in this paper, we propose an energy-efficient and void avoidance depth based routing protocol (EVA-DBR) to address the unique challenges of UWSNs. EVA-DBR proactively detects the void and trapped nodes and bypass them during the routing phase with a passive participation approach. In EVA-DBR, the void and trapped nodes can locally be discovered without imposing any significant overhead, which makes it a simple and scalable approach for void detection and bypassing in UWSNs. By eliminating the need for ACK packets during the updating phase, less energy is consumed by EVR-DBR. It can also adjust its forwarding area according to the density of area ahead which is beneficial to increase the reliability in a sparse area, or reduce the energy consumption resulted from the duplicate packets in a high-density area. The holding time calculation is also further optimised by using the estimated distance and two hops depth information.

The rest of this paper is organised as follows: In Section II, we review the related work in the field. In Section III, we provide a detailed description of the system model. In Section 


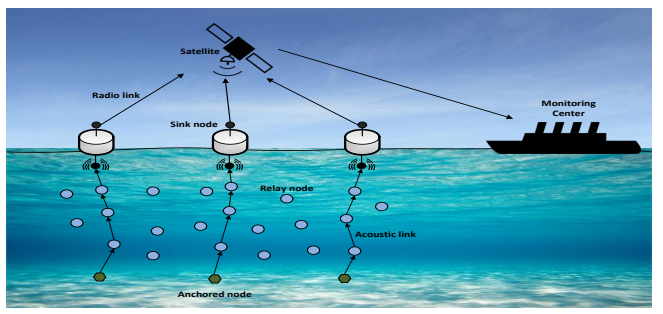

Fig. 1: Network architecture

IV, the details of the EVA-DBR protocol are presented after investigating the void and trapped nodes issue. In Section V, we evaluate the performance of EVA-DBR through simulations. In Section VI, we conclude the paper and discuss future work.

\section{RELATED WORK}

In this section, we review some routing protocols which have been proposed for UWSNs by classifying them into two groups: location-based and depth-based. The main difference between them is related to the location service which is responsible for determining the position of the nodes. The routing protocols in the location-based category assume that underwater nodes are aware of full coordinates of themselves with the aid of some localisation services. In this category, a node is called a void node, if it cannot find any other neighbouring node with the shorter geographical distance to the destination [2].

There is a group of location-based and vector-based routing protocols such as VBF [10], HH-VBF [11], and AHH-VBF [12], which confine the group of forwarding nodes within a virtual pipeline faced toward the only sink on the water surface. However, no solution when facing with a void in the pipeline is provided. VBVA [13] is another vector-based protocol which is equipped with a reactive void handling-technique. VBVA uses two procedures, vector-shift and back-pressure to deal with the convex and concave void, respectively. However, VBVA is very complicated to be adopted in a real underwater environment because it allows a packet be trapped in a concave hole and then be recovered using a time-consuming procedure which increases the end-to-end delay.

Another group of location-based protocols, like GEDAR [14], DCR [15] and GR+DTC [16], exploits a network topology control scheme in which all void nodes can move vertically to be connected to a non-void node. However, topology adjustment of nodes consumes high energy which is only justified when used in the long-term applications.

Depth-based routing protocols are simplified to use only depth information to route the packets, instead of using the full 3D geographical coordinates. In this category, a node is a void node if all of its neighbouring nodes have a higher depth value than itself. DBR [17], and DBMR [18] are depth-based protocols which have not addressed the void problem. In these protocols, each node relays the packet to the neighbouring nodes with lower depth until the packet is delivered to any sink on the surface. HydroCast [3] represents a pressure-based routing protocol which handles the void by using a local lowerdepth-first recovery method. However, the recovery route discovery and maintenance incur high overhead, especially when the recovery path is very long.

There is also a group of pressure-based and soft-state routing protocols like VAPR [4], IVAR [5], and OVAR [6], [7] which can keep the packets away from the void and trapped nodes during the packet forwarding by using the reachability information such as hop count distance and forwarding direction. However, they are not as scalable as a stateless approach. Sometimes changing the status of a node can also alter the status of many other nodes resulting in high overhead.

WDFAD-DBR [19] is another pressure-based routing protocol in which void nodes can take themselves out of the packet forwarding to provide the opportunity for other available candidate nodes. In WDFAD-DBR, the forwarding area is divided into a primary forwarding area (Reuleaux triangle) which is constant all the time, and two auxiliary forwarding areas which may adaptively expand based on the node density and channel quality. However, WDFAD-DBR cannot identify the trapped nodes in advance. As another problem, in WDFAD-DBR, corresponding ACKs in response to each transmitted control packet wastes the network resources. Furthermore, using a fixed primary forwarding area may confine the flexibility of routing to select and modify the forwarding nodes when confronting with different circumstances.

\section{SYSTEM MODEL}

In this section, the network architecture and acoustic propagation model are described in details.

\section{A. Network architecture}

An UWSN has a 3D network topology consisted of anchored, relay and sink nodes, as shown in Fig. 1. In a multisink network model, sink nodes are placed on the water surface as the final destination. They are equipped with both acoustic and radio modems for underwater and land communications, respectively. Anchored nodes are placed at the bottom of the ocean to sense and collect information and deliver it to one of the sinks by using the relay nodes, which are deployed at different depths in between. Then, sink nodes can transmit the collected information to the monitoring centre via satellite for further analysis [10], [11], [17]. The position of relay nodes are continuously changed in the horizontal direction due to the water current: however, this movement is negligible in the vertical direction [12], [19]. It is also assumed that each node knows its current depth by using an embedded pressure gauge. Furthermore, each node can detect Received Signal Strength Indicator (RSSI) which is used to measure the distance between two nodes [19].

\section{B. Acoustic propagation model}

The Thorp model [20] is used for describing the underwater acoustic propagation and transmission models. The path loss or acoustic channel attenuation over distance $d$ is expressed as [20]:

$$
A(d, f)=A_{0} d^{k} \alpha(f)^{d}
$$




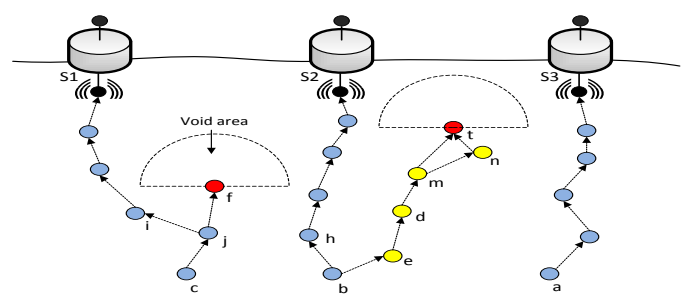

Fig. 2: Void problem in depth-based routings

where $f$ is the signal frequency and $\alpha(f)$ is the absorption coefficient which is defined by the Thorp model. Furthermore, $A_{0}$ represents a unit-normalizing constant, and $k$ is the geometric spreading factor which is set to 1.5 for practical scenarios. The underwater noises are dominant in the different frequency regions and are composed of four main components of turbulence $P N_{t}(f)$, shipping $P N_{s}(f)$, waves $P N_{w}(f)$ and thermal energy $P N_{t h}(f)$ which can be expressed as [7], [12]:

$$
P N(f)=P N_{t}(f)+P N_{s}(f)+P N_{w}(f)+P N_{t h}(f)
$$

By considering the attenuation and underwater environment noise models, the signal-to-noise ratio (SNR) over distance $d$ with the signal frequency $f$ can be expressed as [20]:

$$
S N R(d, f)=\frac{P R(f)}{A(d, f) P N(f)}
$$

where $P R(f)$ represents the transmission power with frequency $f$ at the forwarding node. Decoding of the received packet can be done when $S N R$ at the receiver is greater than the detection threshold.

\section{EVA-DBR ROUTING PROTOCOL}

In this section, our proposed routing protocol, called Energy-efficient and Void Avoidance Depth Based Routing (EVA-DBR), is presented. In EVA-DBR, in addition to detecting the void nodes, the trapped nodes can also be locally detected and then become inactive in the routing phase to provide more chance for other regular nodes to forward the data packets. Furthermore, periodical updating phase is designed to send an updating packet in each updating interval without the need to receive any ACK from the neighbouring nodes. The updating packets are used to keep updating the nodes about the neighbouring nodes status. Finally, by using an adaptive forwarding area, the aims of suppressing the duplicate packets in a dense density, or including a greater number of candidate nodes in a sparse density, is achieved.

\section{A. Problem description}

In the category of depth-based routing protocols, each forwarding node forwards the packets to the neighbouring nodes with the lower depth than itself. The neighbouring nodes with lower distance from the surface, have higher priority to forward the packet [3]. According to our routing criteria, an underwater sensor node is called a regular node when it has access to a neighbouring node with lower depth than itself. For example, node $c$ in Fig. 2 is a regular node because it can see another regular node $j$ in its neighbourhood with the lower depth.

Unlike a regular node, a void node is defined as an underwater node which cannot find any qualified node with a lower depth towards the water surface. The packets reached at a void node must be dropped in a depth-based routing protocol without a void handling mechanism [3]-[5]. For instance, nodes $f$ and $t$ are the void nodes since there exist no nodes above them. Thus, in a greedy depth-based forwarding like DBR [17], when a void node is considered as one of the candidate nodes, it obtains higher priority to forward the packet since it has lower depth than other candidate nodes. Subsequently, transmission by a void node may suppress other candidate nodes from forwarding the packet, resulting in the packet dropping.

Additionally, there is another kind of nodes which can see any other node above; however, forwarding packets to these nodes eventually leads the packets to get stuck in a void node. These group of nodes are called the trapped nodes [4], [7]. For example, if node $b$ forwards a packet to node $e$, instead of node $h$, the packet is eventually stuck in node $t$. The routing efficiency can obviously be increased if the trapped nodes are also exempted from the packet forwarding. Our proposed routing protocol is, to the best of our knowledge, the first stateless routing protocol which is also able to bypass the trapped nodes.

\section{B. Overview of EVA-DBR protocol}

EVA-DBR is a stateless routing protocol which only relies on the information received from one-hop neighbours for void detection and bypassing in the routing phase. Initially, all nodes are considered as regular nodes in the network. However, over time, void and trapped nodes are detected using the received information from the neighbouring nodes.

In the routing phase, in a passive participation manner, all void and trapped nodes remove themselves from the packet forwarding candidates, providing an opportunity for the regular nodes to increase the packet delivery probability in each transmission. In a sparse region, EVA-DBR may let the duplicated transmissions to increase the packet delivery probability; however, in a dense area, the forwarding area is limited to suppress the duplicate packets. Furthermore, EVA-DBR takes advantage of the relative distance and two hops depth information to further optimise the holding time calculation. Algorithm 1 details the two phases of EVA-DBR: updating phase and routing phase.

\section{Updating phase}

In EVA-DBR, each regular node periodically broadcasts a control packet including the packet type, node ID, node type, and node depth to inform the neighbouring nodes about its current status. Each node keeps a neighbouring table to maintain the required information about its neighbouring nodes. Upon receiving a control packet, each receiving node updates its neighbouring table based on the received control packets. Each receiving node also sets an invalidation timer for a neighbouring node after receiving a control or data packet. The invalidation timer is reset upon reception of new information from the node. Otherwise, the neighbouring node 


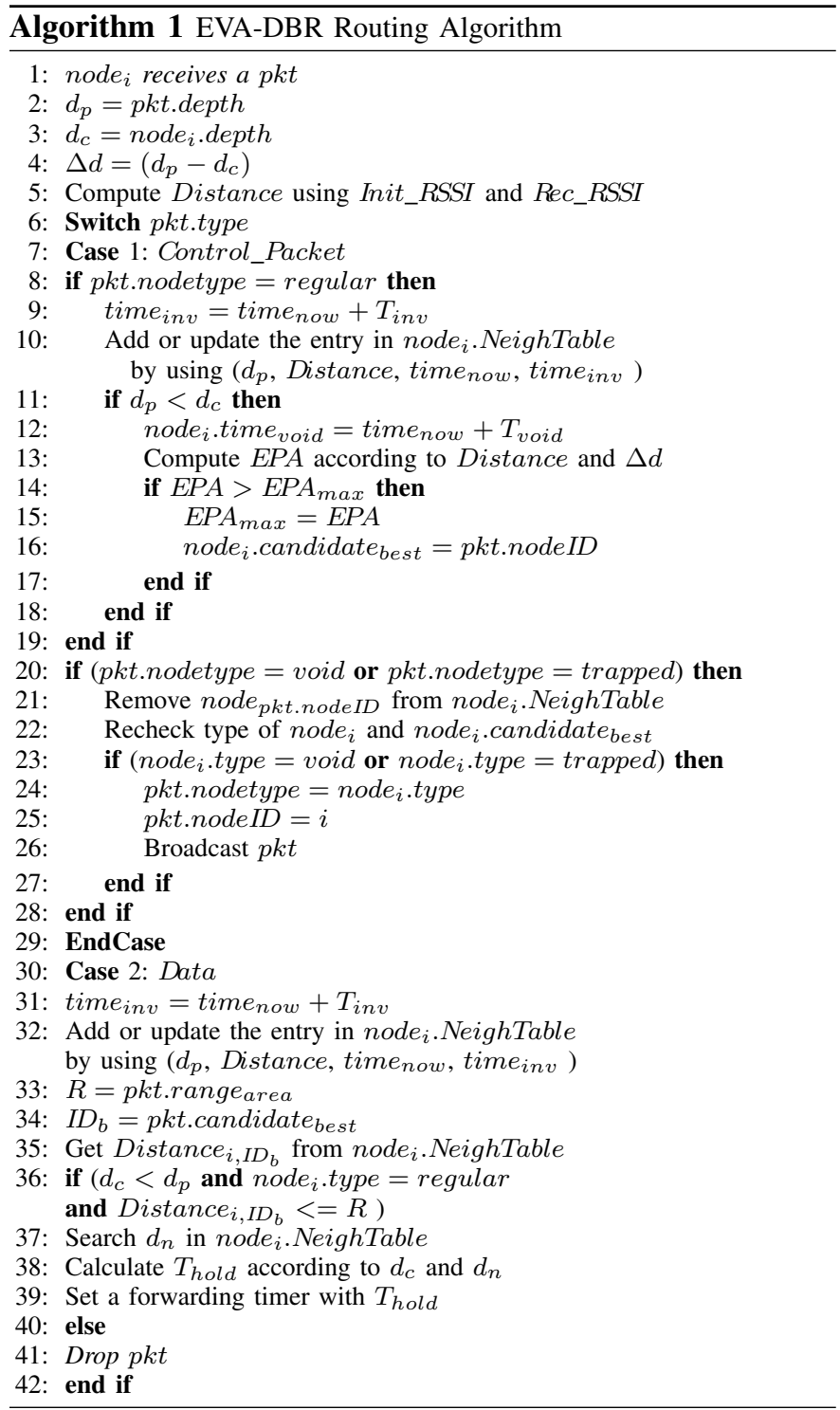

is removed from the neighbouring table when the invalidation timer is expired.

Each entry of neighbouring table contains the neighbouring node ID and depth, the distance between the current node and the neighbouring node, time of receiving the packet, and invalidation time of the neighbouring node. The relative distance between each pair of nodes can be computed via the difference between RSSI of initial and received signals [19]. If all the nodes are homogeneous in terms of the transmission power, the initial RSSI would be known to each node. By using the Thorp propagation model [20], each node computes its distance to all neighbouring nodes and keeps it at the neighbouring table.

For the updating phase, there are some issues which should be addressed properly to increase the network performance. First, broadcasting a control packet at the same time by regular nodes may result in collisions in the network. Thus, in order to prevent the collision problem, each node selects its transmission time randomly from a particular interval. Second, if the updating operation is carried out very frequently, it may lead to increase in energy consumption. Thus, the updating phase period should be carefully specified depending mainly on the water current speed. Moreover, in our model, the nodes are exempt from sending back any ACK to the sender node which leads to more energy saving.

In EVA-DBR, void node detection can be performed in a timer-based approach. In this way, each node can set a voiddetection timer upon starting its operation. During this time, the node waits for a packet from the neighbouring nodes with lower depth. Upon receiving a control or data packet from a neighbouring node with the lower depth, receiving node resets its void-detection timer. If no neighbouring node with lower pressure is sensed before the expiration of the void detection timer, node announces itself as a void node by broadcasting a control packet without any delay for its neighbours which are located below. Sometimes nodes have already received some control packets from other nodes with lower depth; however, over time, topology is changed, and they may become a void node which again can be detected using the void-detection timer. A void detection can be announced immediately because void nodes are not in the transmission range of each other, hence, there is no chance of collision. For example, if another void node places above node $t$ in Fig. 2, it is no longer a void node but becomes a trapped node for this newcomer void node. The void-detection timer should be long enough and more than the maximum range of the updating timer to have a reliable outcome for the void and trapped node detection.

Furthermore, trapped nodes can be detected by an eventdriven approach. Upon receiving a control packet from a void node, each node updates its neighbouring table and checks whether it still has any regular neighbour with lower depth than itself or not. If the void node is the only neighbouring node with the lower depth in the neighbourhood, in a similar way, receiving node announces itself as a trapped node by broadcasting a control packet including its ID and its current status. Similarly, when a node receives a control packet from a trapped node, it updates the status of the trapped node in the neighbouring table and checks its current status. If its neighbouring table does not include any node with lower depth except the trapped node, it also marks itself as a trapped node and broadcast a control packet to inform other nodes. This procedure stops when all trapped nodes in a local area are detected. In this way, all void and trapped nodes in different places of the network topology can locally be identified without needing to know the network topology.

During the updating phase, each regular node also selects the best candidate node in terms of the Expected Packet Advancement (EPA) among the neighbouring nodes with lower depth, to be used as a reference node in the opportunistic data forwarding. The expected packet advancement of each neighbouring node can be measured by $P \times \Delta d$, where $\Delta d$ is the depth difference between the forwarding node and receiving node, and $P$ is the packet error probability over the distance between these two nodes. The bit error probability over distance $d$ can be computed as follows [3]:

$$
P_{e}(d)=\frac{1}{2}\left(1-\sqrt{\frac{S N R_{a v g}(d, f)}{1+S N R_{a v g}(d, f)}}\right)
$$


where $S N R_{a v g}(d, f)$ is the average signal-to-noise ratio over distance $d$ with frequency $f$ which can be calculated using Eq. 3. Accordingly, the delivery probability of a data packet with size $n$ bits over distance $d$ can be expressed as follows [3]:

$$
P=\left(1-P_{e}(d)\right)^{n}
$$

The packet delivery probability has an inverse relationship with the traversed distance. Thus, a neighbouring node with the lowest depth may not necessarily be a qualified node for relaying the packet due to its less chance to receive the data packet without any error. In order to find the best candidate node, upon reception of a control packet, each receiving node computes the EPA of the sending node and updates its best candidate node ID if it is required.

\section{Routing phase}

The aim of the routing phase is to deliver a data packet to one of the sinks on the water surface. To accomplish a successful delivery, each packet is required to be delivered successfully at each hop towards one of the destinations. Thus, to demonstrate the routing phase, we investigate the packet forwarding at each hop.

Due to high bit error rate, each forwarding node should take advantage of a group of candidate nodes at each hop to successfully relay the data packet [9]. Having only one candidate node to forward the packet may lead to the energy wastes, due to increasing in the number of retransmissions resulted from the high bit error probability [7]. At the other extreme, if all receiving nodes participate in the packet forwarding, it is a purely flooding which also wastes the network resources. Thus, an efficient routing protocol should employ some constraints to limit the number of participating nodes to obtain an ideal forwarding set in terms of expected packet advancement [21]. Moreover, during the routing phase, each node also can update the neighbouring table according to the information extracted from the data packet header. This feature aids to obtain fresh information about the neighbouring nodes and to extend the updating phase duration. Data header includes the packet type, node ID, node depth, the sequence number of the packet, the range of the forwarding area, and ID of the best candidate node.

Forwarding node selection criteria: In EVA-DBR, when a node receives a data packet, it first updates its neighbouring table and then checks its eligibility to whether participate in the packet forwarding or not. If it is not an eligible candidate node for packet forwarding, it only drops the received packet. For more energy saving, the nodes only read the header of the packet for early acceptance or rejection. In order to be placed among the candidate nodes, the receiving node should satisfy some conditions. First, the receiving node should have lower depth and be placed at the upper hemisphere of the forwarding node transmission coverage area. Second, the receiving node should be a regular node, not a trapped or void node. The trapped and void nodes which have already been detected, only drop the received packet. Third, the receiving node should be placed within the forwarding region which requires that its relative distance to the best candidate node is smaller than a certain value. The receiving nodes outside of this

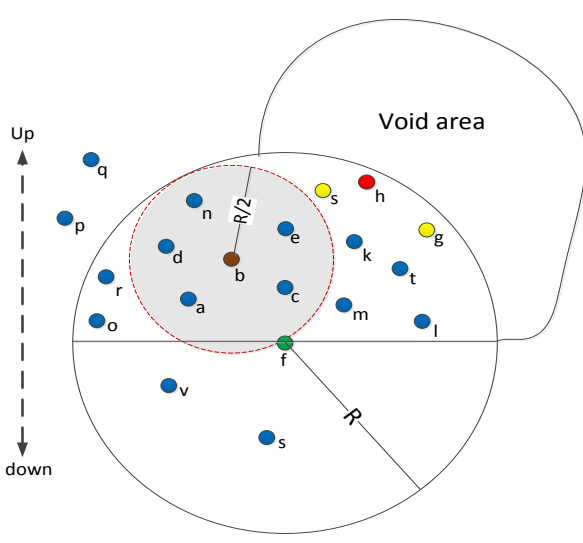

Fig. 3: Forwarding area in EVA-DBR

area simply drop the received packets since they are located within the suppression region. For instance, in Fig. 3, only the neighbouring nodes $a, c, d, e, n$ along with the best candidate node $b$, participate in the packet forwarding.

Forwarding area features: The forwarding area can be resized dynamically, while it has a significant impact on the duplicate packets suppression in a dense area and also on the reliability enhancement in a sparse area. At each hop, each forwarding node can select a forwarding range according to the density of the area ahead. The number of nodes with the lower depth can be extracted from the neighbouring table to adopt the forwarding area size. If the number of regular nodes with lower depth is more than a maximum threshold, the minimum forwarding range, which is set to $R / 4$ in our model, can be selected to store in the data packet header to suppress the largest possible number of duplicate transmissions. On the contrary, if the number of regular nodes with lower depth is less than a minimum threshold, the maximum forwarding range, which is set to $R$ in our model, can be adjusted to increase the reliability of packet transmission. In a similar way, if the area ahead density varies from the minimum to the maximum threshold, the forwarding range can also be selected within the interval $[R / 4, R]$. It should be noted that there is no hidden node within the forwarding node set if the forwarding range is set less than $R / 2$ since all nodes are placed within the transmission range of each other.

Holding time calculation: Eventually, all eligible candidate nodes should set a forwarding timer for packet transmission. In EVA-DBR, the forwarding time is computed using the two hops advancement. Upon receipt of a data packet, each candidate node $\left(c_{n}\right)$ searches its neighbouring table to find a node with the lowest depth $\left(c_{n+1}\right)$ and then calculates the holding time based on the depth difference between the previous hop forwarding node $\left(c_{n-1}\right)$ and this next-hop neighbouring node $\left(c_{n+1}\right)$. It then updates the two fields of the depth and ID of the best candidate node in the date packet header and sets a forwarding timer according to the holding time. If the forwarding timer expires and another transmission of this packet is not yet heard, it transmits the packet; otherwise, it drops the packet. 
The holding time which is used to calculate the forwarding time should satisfy some conditions to assure priority-based scheduling of the candidate nodes and also suppressing the duplicate packets. First, the holding time should decrease with the increase of the depth difference from the forwarding node. Second, the holding time should be long enough to let other candidate nodes to hear the packet transmission before forwarding the same packet. In EVA-DBR, each candidate node calculates its holding time, $T_{\text {hold }}$, using:

$$
T_{\text {hold }}=\frac{(R-H)}{R} T_{\text {Delay }}+\frac{R-D}{\nu_{\text {sound }}}
$$

where $R$ and $\nu_{\text {sound }}$ are the transmission range of the node, and the propagation speed of sound in the water, respectively. $D$ is the relative distance between the forwarding node to the candidate node which can be calculated based on the difference between initial RSSI and received RSSI of the transmission signal [19]. $H$ is the depth difference sum of two hops which can be calculated by $H=\alpha h_{i}+(1-\alpha) h_{i+1}$ where $h_{i}$ is the depth difference from the forwarding node to the candidate node and $h_{i+1}$ is the depth difference from the candidate node to the next hop candidate node with the lowest depth and $\alpha$ is the weighting coefficient which is within the interval $[0,1] . T_{\text {Delay }}$ is the predefined maximum delay which should be long enough to be able to suppress the transmission of lower priority nodes before relaying the packet.

The first part of Equation 6 is considered to ensure the priority among the candidate nodes for packet forwarding based on the two hops advancement and, the second part of the equation is intended to compensate the propagation delays from the forwarding node to all candidate nodes. If the fixed value of $T_{\text {Delay }}$ is set high, the holding time of packets increases which eventually leads longer end-to-end delay and less transmission reliability; however, it contributes to more energy saving since a greater number of redundant packets can be suppressed. In contrast, if $T_{\text {Delay }}$ is set low, the endto-end delay and packet failure rate decrease; however, energy consumption increases due to the inability to suppress some lower priority nodes. Therefore, there is a tradeoff between the energy consumption and latency in determining the amount of $T_{\text {Delay }}$ which can be set according to the performance objectives during the network operation.

\section{EXPERIMENTAL RESULTS}

In this section, the details of our simulation study and also the performance results are presented using Aqua-Sim, an NS2 based simulating software for underwater acoustic networks, to evaluate EVA-DBR against DBR, and WDFAD-DBR in a multi-sink architecture.

\section{A. Simulation setup}

In the simulation, we use CSMA MAC protocol without using its RTS/CTS and ACK mechanism to offset the effects of high propagation delay in the underwater environment. When a forwarding node senses channel is free, it forwards the packet; otherwise, it will back off. After five times back-off, the forwarding node discards the data packet. The nodes need to listen to the channel continuously to suppress any duplicate packet which is already relayed by another candidate node.
The underwater acoustic communication channel described in Section III is used in our simulation. We deploy the relay nodes (ranging from 400 to 800 ) randomly in a $500 \mathrm{~m} \times$ $500 m \times 1000 m$ 3D field which makes it a sparse and deep network. The transmission power and the receiving power threshold for a packet are set to $90 d B$ re $\mu \mathrm{Pa}$ and 10 $d B$ re $\mu P a$, respectively. Each node also consumes $2 \mathrm{~W}$ and $0.75 W$ energy for sending and receiving a packet, respectively, while the idle power consumption is equal to 8 $m W$. The transmission of nodes is considered as 100 meters. Each node generates a data packet every 1 seconds which is long enough to prevent the interference of two continuous packets. The bit rate is set to $10 \mathrm{kbps}$. The value of weighting coefficient $\alpha$ is set to 0.6 to make the routing decision more dependent on the current node depth, to obtain a forwarding area with more unvisited candidate nodes, which can improve the reliability of routing. The $T_{\text {Delay }}$ in Eq. 6 is set as $2 R / \nu_{\text {sound }}$. Following a Random Walk 2D mobility model, relay nodes move horizontally (in the X-Y plane) at the speed of $2 \mathrm{~m} / \mathrm{s}$. The source node is randomly placed at the bottom of the ocean with depth $1000 \mathrm{~m}$ and its position is fixed by the end of each simulation run. Furthermore, we consider 5 sink nodes to collect the information at locations $(250 ; 250 ; 0),(125 ; 125$; $0),(125 ; 375 ; 0),(375 ; 125 ; 0),(375 ; 375 ; 0)$. The data packet size is fixed at 50 Byte, because at each hop, only the ID of the best candidate node is included in the data packet header, not all forwarding candidate nodes IDs. The acoustic signal propagation speed is set to $1500 \mathrm{~m} / \mathrm{s}$ for a deep underwater environment. The updating interval varies randomly in a range from $T_{\text {update }}$ to $\left(T_{\text {update }}+T_{\text {update }} \times 20 \%\right)$ where $T_{\text {update }}$ is set to $50 \mathrm{~s}$. The node invalidation time, $T_{\text {invalid }}$, and node void detection timer, $T_{\text {void }}$, are also considered as 60 seconds. All the results are averaged over 25 runs for randomly generated topologies while the simulation time for each round is set to 1000 seconds.

\section{B. Results and Analysis}

In this section, we assess the performance of EVA-DBR against those of DBR, and WDFAD-DBR in terms of packet delivery ratio, energy tax, end-to-end delay, forwarding number, and traversed distance.

Packet Delivery Ratio (PDR): It is defined as the ratio of the number of packets successfully received by the sink nodes to the number of packets sent by the source node. The results for the packet delivery ratio in different node density are shown in Fig. 4. For all these protocols, PDR increases by increasing the number of nodes, since the network topology becomes more connected which reduces the probability of void occurrence.

As can be seen, EVA-DBR always has higher PDR than those of DBR and WDFAD-DBR. It is because EVA-DBR can exclude all the routes leading to a trapped or void area, and give the chance of packet delivery to the regular nodes. On the other hand, DBR does not have any void-handling technique, and there is also no mechanism for the trapped area avoidance in WDFAD-DBR. Furthermore, EVA-DBR can adjust the size of forwarding area according to the node density of area ahead which increases the chance of packet delivery by including more candidate nodes when density is low or 


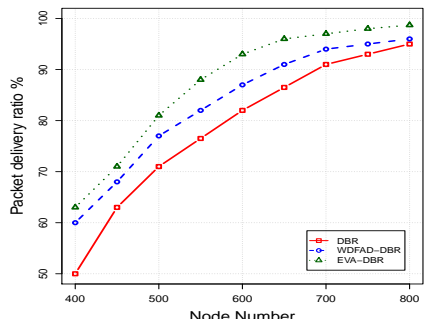

Fig. 4: Packet delivery ratio vs. node density

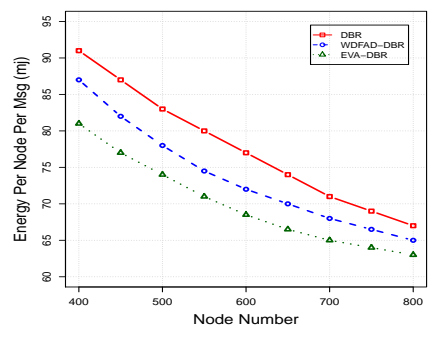

Fig. 5: Energy consumption per message vs node density

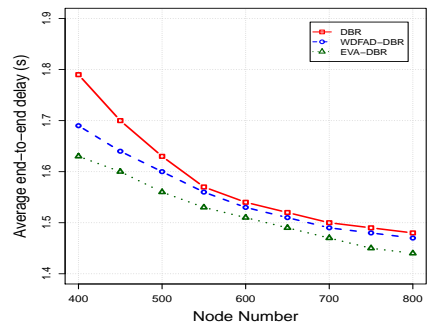

Fig. 6: Average end-to-end delay vs node density by reducing the number of collisions when density is high. Finally, the updating phase in EVA-DBR can be performed more frequently which can provide the fresher information for the packet forwarding. However, due to high overhead of WDFAD-DBR in sending and receiving the ACK packets, the updating phase should be performed less frequently leading to performance reduction for packet delivery.

Energy tax: The energy tax is measured in millijoule (mj) in terms of the average energy consumed per node and per message to successfully deliver a packet to a sink node. The energy tax of routing protocols in different node density are shown in Fig. 5. As can be seen, the energy tax of routing protocols intends to decrease with the increase of node number, as more data packets can successfully be delivered in a well connected dense network.

EVA-DBR has the lowest energy consumption among the routing protocols. First of all, in EVA-DBR, when a forwarding node encounters a dense area, its forwarding area size and position can be adjusted to suppress the redundant packet transmissions. However, in a dense network, many nodes may be placed in the upper hemisphere of DBR and also in the fixed primary area of WDFAD-DBR (auxiliary area) leading to increase in the number of packet receptions, transmissions, and collisions. Second, in a sparse density for DBR and WDFAD-DBR, many packets are dropped due to get stuck in the trapped and void area resulting in more energy waste. However, EVA-DBR can reduce the energy waste of stuck packets by excluding the routes leading to the void nodes. Third, the updating phase in EVA-DBR is performed at significantly lower energy cost compared to WDFAD-DBR, by eliminating the need for ACK packets.

End-to-end delay: It is defined as the average delay time taken from the moment of the creation of packets at the source node until successfully being delivered to the sink node. In a multi-sink architecture, due to the fact that a packet may be delivered to different sink nodes at different times, the shortest end-to-end delay is used in the simulation results. As can be observed from Fig. 6, the end-to-end delay for each protocol decreases with the increase of node number because each forwarding node can find more qualified nodes with greater advancement toward the surface when the network is dense. The nodes with more advancement also have shorter holding time which reduces the total holding time of the packet.

As shown in Fig. 6, the latency of EVA-DBR is lower than other protocols. First of all, EVA-DBR and WDFAD-
DBR take advantage of two hop advancements to calculate the holding time, but DBR uses only one hop advancement. Second, as the traversed distance and hop number for EVADBR are lower than other protocols, its end-to-end delay also becomes less compared to other protocols. Third, in EVADBR, the candidate nodes with higher expected packet advancement are selected without regarding their locations which are beneficial to reduce the latency; however, in WDFADDBR, most of the packets forwarding are limited within a fixed primary forwarding region which reduces the flexibility and consequently increases the latency. Fourth, the holding time calculation in EVA-DBR is more optimised compared to WDFAD-DBR, because EVA-DBR takes into account the exact value of propagation delay from the forwarding node to all candidate nodes and not only the worst case value for the holding time calculation, as it can be observed from the second part of Eq. 6. Finally, in EVA-DBR, the number of collisions and retransmissions reach the least amount possible which is useful to reduce the packet delivery time.

Forwarding number: It shows the average number of transmissions by each node during the simulation time. The forwarding number has a considerable impact on the energy expenditure and number of packet collisions. The results for the forwarding number is shown in Fig. 7.

The forwarding number in EVA-DBR is always lower than other protocols. First of all, EVA-DBR has more flexibility to resize and place the forwarding area in a particular position. However, DBR uses all neighbouring nodes with lower depth at each hop, and WDFAD-DBR only can resize its auxiliary areas and not its primary forwarding area. Second, EVA-DBR can also suppress the packet transmissions from all void and trapped nodes. However, DBR cannot deprive the void and trapped nodes from packet forwarding and WDFAD-DBR can only prevent the void nodes from the packet transmissions and not the trapped nodes. Third, finding the shortest path for each data packet towards a destination in EVA-DBR also has the contribution in the reduction of forwarding number in comparison to DBR and WDFAD-DBR.

Traversed distance: The traversed distance is defined as the average total traversed distance by each packet from the source to the sink node. Due to the fact that each packet may be delivered to different sinks with various paths, the shortest traversed path is used in the simulation results. The traversed distance has an impact on other parameters, in particular on the amount of end-to-end delay. The results for traversed distance 


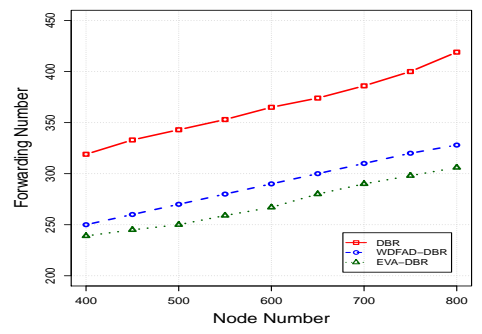

Fig. 7: Forwarding number vs node density

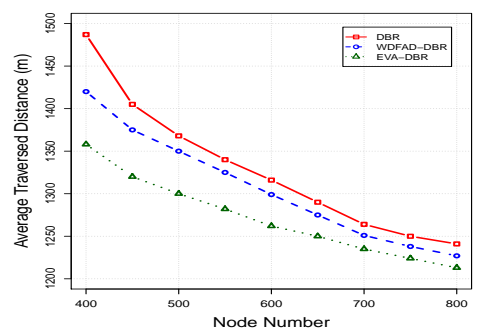

Fig. 8: Average traversed distance vs node density

is shown in Fig. 8. As can be observed, the average traversed distance decreases by increasing the node density. In sparse scenarios, the shortest path to the sink is not always covered by some nodes, and consequently, a longer path may be taken to reach the destination. On the other hand, in dense scenarios, the chance of finding the shorter routes is higher.

EVA-DBR is able to find the shorter routes for delivering the packets in comparison to other protocols. First of all, considering the two hop advancements in WDFAD-DBR and EVA-DBR contributes to finding the shorter routing path compared to DBR, which uses only one hop advancement. Second, EVA-DBR has the flexibility to place its forwarding area everywhere to cover more candidate nodes with greater advancement; however, other protocols are deprived of such ability.

\section{CONCLUSION AND OPEN RESEARCH ISSUES}

The void communication problem is considered as one of the most challenging factors when designing routing protocols. In this paper, we have proposed EVA-DBR, a depth based and stateless routing protocol which can bypass both void and trapped areas by applying a preventive void-handling technique. EVA-DBR exploits the local information obtained from the updating phase, detects the void and trapped nodes to exclude them from the packet forwarding candidates set, adjusts the forwarding area based on the network density and, finally, calculates the holding time for each forwarding node based on the energy-reliability trade-off constraints. The simulation results have demonstrated that EVA-DBR significantly decreases packet loss, energy consumption, end-to-end delay, forwarding number and traversed distance in sparse to dense scenarios. As future work, we plan to design an efficient underwater MAC protocol for EVA-DBR to improve the network performance in terms of reducing the number of collisions.

\section{REFERENCES}

[1] I. F. Akyildiz, D. Pompili, and T. Melodia, "Underwater acoustic sensor networks: research challenges," Ad hoc networks, vol. 3, no. 3, pp. 257 279, 2005.

[2] M. T. Kheirabadi and M. M. Mohamad, "Greedy routing in underwater acoustic sensor networks: a survey," International Journal of Distributed Sensor Networks, vol. 2013, 2013.

[3] U. Lee, P. Wang, Y. Noh, F. Vieira, M. Gerla, and J.-H. Cui, "Pressure routing for underwater sensor networks," in INFOCOM, 2010 Proceedings IEEE, pp. 1-9, IEEE, 2010.

[4] Y. Noh, U. Lee, P. Wang, B. S. C. Choi, and M. Gerla, "Vapr: void-aware pressure routing for underwater sensor networks," Mobile Computing, IEEE Transactions on, vol. 12, no. 5, pp. 895-908, 2013.

[5] S. M. Ghoreyshi, A. Shahrabi, and T. Boutaleb, "An inherently void avoidance routing protocol for underwater sensor networks," in Twelfth International Symposium on Wireless Communication Systems, IEEE, 2016.

[6] S. M. Ghoreyshi, A. Shahrabi, and T. Boutaleb, "An opportunistic void avoidance routing protocol for underwater sensor networks," in IEEE 30th International Conference on Advanced Information Networking and Applications (AINA), pp. 316-323, March 2016.

[7] S. M. Ghoreyshi, A. Shahrabi, and T. Boutaleb, "A novel cooperative opportunistic routing scheme for underwater sensor networks," Sensors, vol. 16 , no. 3 , p. 297,2016

[8] D. Chen and P. K. Varshney, "A survey of void handling techniques for geographic routing in wireless networks," Communications Surveys \& Tutorials, IEEE, vol. 9, no. 1, pp. 50-67, 2007.

[9] A. Darehshoorzadeh and A. Boukerche, "Underwater sensor networks: a new challenge for opportunistic routing protocols," Communications Magazine, IEEE, vol. 53, no. 11, pp. 98-107, 2015.

[10] P. Xie, J.-H. Cui, and L. Lao, "Vbf: vector-based forwarding protocol for underwater sensor networks," in Networking 2006. Networking technologies, services, and protocols; performance of computer and communication networks; mobile and wireless communications systems, pp. 1216-1221, Springer, 2006.

[11] N. Nicolaou, A. See, P. Xie, J.-H. Cui, and D. Maggiorini, "Improving the robustness of location-based routing for underwater sensor networks," in OCEANS 2007-Europe, pp. 1-6, IEEE, 2007.

[12] H. Yu, N. Yao, and J. Liu, "An adaptive routing protocol in underwater sparse acoustic sensor networks," Ad Hoc Networks, 2014.

[13] P. Xie, Z. Zhou, Z. Peng, J.-H. Cui, and Z. Shi, "Void avoidance in three-dimensional mobile underwater sensor networks," in Wireless Algorithms, Systems, and Applications, pp. 305-314, Springer, 2009.

[14] R. W. Coutinho, A. Boukerche, L. F. Vieira, and A. A. Loureiro, "Gedar: geographic and opportunistic routing protocol with depth adjustment for mobile underwater sensor networks," in Communications (ICC), 2014 IEEE International Conference on, pp. 251-256, IEEE, 2014.

[15] R. W. Coutinho, L. F. Vieira, A. Loureiro, et al., "Dcr: Depth-controlled routing protocol for underwater sensor networks," in Computers and Communications (ISCC), 2013 IEEE Symposium on, pp. 000453 000458, IEEE, 2013.

[16] R. W. Coutinho, A. Boukerche, L. F. Vieira, and A. A. Loureiro, "A novel void node recovery paradigm for long-term underwater sensor networks," Ad Hoc Networks, 2015.

[17] H. Yan, Z. J. Shi, and J.-H. Cui, "Dbr: depth-based routing for underwater sensor networks," in NETWORKING 2008 Ad Hoc and Sensor Networks, Wireless Networks, Next Generation Internet, pp. 72 86, Springer, 2008.

[18] L. Guangzhong and L. Zhibin, "Depth-based multi-hop routing protocol for underwater sensor network," in Industrial Mechatronics and Automation (ICIMA), 2010 2nd International Conference on, vol. 2, pp. 268270, IEEE, 2010.

[19] H. Yu, N. Yao, T. Wang, G. Li, Z. Gao, and G. Tan, "Wdfad-dbr: Weighting depth and forwarding area division dbr routing protocol for uasns," Ad Hoc Networks, vol. 37, pp. 256-282, 2016.

[20] M. Stojanovic, "On the relationship between capacity and distance in an underwater acoustic communication channel," ACM SIGMOBILE Mobile Computing and Communications Review, vol. 11, no. 4, pp. 34 43, 2007.

[21] K. Zeng, W. Lou, J. Yang, and D. R. Brown, "On geographic collaborative forwarding in wireless ad hoc and sensor networks," in Wireless Algorithms, Systems and Applications, 2007. WASA 2007. International Conference on, pp. 11-18, IEEE, 2007. 